\title{
Investigation on Alternobaric Vertigo of Gradational Simulated Saturation Diving with the Deepest Saturation of $480 \mathrm{~m}$ to $-493 \mathrm{~m}$
}

\author{
Jianguang Zhou ${ }^{1, *}$, Changyun Liu², Yingqi Zhou ${ }^{1}$ \\ ${ }^{1}$ Naval Center for Specialty Medicine, Shanghai, China \\ ${ }^{2}$ Hongkou District of Changhai Hospital Affiliated to Naval Medical University, Shanghai, China
}

\section{Email address:}

zhoujg411@aliyun.com (Jianguang Zhou), Liucy21xh@126.com (Changyun Liu), zhouyingqi81@163.com (Yingqi Zhou)

${ }^{*}$ Corresponding author

\section{To cite this article:}

Jianguang Zhou, Changyun Liu, Yingqi Zhou. Investigation on Alternobaric Vertigo of Gradational Simulated Saturation Diving with the Deepest Saturation of 480m to -493m. Science Discovery. Vol. 9, No. 1, 2021, pp. 27-31. doi: 10.11648/j.sd.20210901.15

Received: February 23, 2021; Accepted: March 8, 2021; Published: March 12, 2021

\begin{abstract}
Objective To provide medical support for deep helium-oxygen saturation diving. Methods According to the data, the diagnostic criteria of Alternobaric vertigo were established. Gradational simulated saturation diving at $65 \mathrm{~m}, 250 \mathrm{~m}$ and $480 \mathrm{~m}$ helium-oxygen saturation $-493 \mathrm{~m}$ (hereinafter referred to as $480 \mathrm{~m}$ ) in $500 \mathrm{~m}$ saturation system, questionnaire, physical examination, Nystagmus view detector and Magnetic resonance imaging were used to check divers before and after each pressure exposure. Results 1 person at $250 \mathrm{~m}$ and $480 \mathrm{~m}$ after leaving the cabin each had hyposmia, and $1 \mathrm{e}$ person at $250 \mathrm{~m}$ and $480 \mathrm{~m}$ after leaving the cabin each had knee reflex $(+)$, but the two sides were symmetrical. 1 person at $250 \mathrm{~m}$ and $480 \mathrm{~m}$ after leaving the cabin each had finger nose test $(+)$, and 1 person at $480 \mathrm{~m}$ after leaving the cabin had heel-knee-tibia test $(+) .1$ person at $65 \mathrm{~m}, 250 \mathrm{~m}$ and $480 \mathrm{~m}$ after leaving the cabin each had hyperemia in the eardrum, and 1 person at $250 \mathrm{~m}$ and 2 people at $480 \mathrm{~m}$ after leaving the cabin had caloric test $(+)$, all on the right side. 2 people at $250 \mathrm{~m}$ and $480 \mathrm{~m}$ after leaving the cabin each were found to be impaired by electrical audiometry. All the abnormal people above were reexamined normal the next day. When the pressure changed, there were 5 person-times of dizziness (13s on average), 4 person-times of aural fullness (177.5s on average), 7 person-times of shaking sensation $(337.14 \mathrm{~s}$ on average), 5 person-times of nausea ( $312 \mathrm{~s}$ on average), 3 person-times of dizziness (15min on average), 1 person-time of headache in total (20s), 6 person-times of Romberg and 2 person-times of Fukuda in total. Alternobaric vertigo was diagnosed 1 person-time and most likely 1 person-time at $65 \mathrm{~m}, 2$ person-times and most likely 2 person-times at $250 \mathrm{~m}$ and 3 person-times at $480 \mathrm{~m}$. Conclusion Alternobaric vertigo occurs frequently in deep helium-oxygen saturated diving, which is characterized by short time, complete reversibility and no influence, but should be paid attention to.
\end{abstract}

Keywords: Helium-oxygen, Saturated Diving, Divers, Alternobaric Vertigo

\section{深度递增模拟氦氧最深480m饱和-439m巡回潜水变压性眩晕的调}

\section{查}

周建光 $^{1^{*}}$, 刘长云 ${ }^{2}$, 周颖奇 ${ }^{1}$

${ }^{1}$ 海军特色医学中心, 上海, 中国

2海军军医大学附属长海医院虹口院区, 上海, 中国

邮箱

zhoujg411@aliyun.com（周建光）, Liucy21xh@126.com（刘长云），zhouyingqi81@163.Com（周颖奇） 
摘要: 目的: 为大深度氦氧饱和潜水提供医学保障。方法制定变压性眩晕诊断标准, 在 $500 \mathrm{~m}$ 饱和系统进行模拟 $65 \mathrm{~m}$ 、 $250 \mathrm{~m} 、 480 \mathrm{~m}$ 氦氧饱和 $-493 \mathrm{~m}$ （以下简称 $480 \mathrm{~m}$ ）巡回潜水实验中，采用问卷、查体、眼震视图仪、核磁共振等对潜水员 入舱前、出舱后进行检查。结果: $250 \mathrm{~m} 、 480 \mathrm{~m}$ 出舱后各有 1 人嗅觉减退，各有 1 人指鼻试验 $(+)$, 各有 1 人膝反射 $(+)$, 但是双侧对称， $480 \mathrm{~m}$ 出舱后有 1 人跟胫膝实验 $(+) ， 65 \mathrm{~m} 、 250 \mathrm{~m} 、 480 \mathrm{~m}$ 出舱后各有 1 人鼓膜充血， $250 \mathrm{~m}$ 出舱后 1 人， 480 出舱后 2 人冷热实验 (+) , 均右侧, $250 \mathrm{~m} 、 480 \mathrm{~m}$ 出舱后各有 2 人电测听示感音性受损, 以上所有异常者第二天复 查均正常。压力变化时天旋地转 5 人次, 平均 $13 \mathrm{~S}$, 耳闷 4 人次, 平均 $177.5 \mathrm{~s}$, 摇晃感 7 人次, 平均 $337.14 \mathrm{~s}$, 恶心 5 人次, 平均 $312 \mathrm{~s}$, 头昏 3 人次, 平均 $15 \mathrm{~min}$, 头痛 1 人次, $20 \mathrm{~s}$, Romberg6人次, Fukuda 2 人次。变压性眩晕 $65 \mathrm{~m}$ 确诊 1 人次, 极 可能 1 人次, $250 \mathrm{~m}$ 确诊 2 人次, 极可能 2 人次, $480 \mathrm{~m}$ 确诊 3 人次。结论: 大深度氦氧饱和潜水变压性眩晕多人次发生, 特点是时间短, 完全可逆, 但是应引起重视。

关键词: 氦氧, 饱和潜水, 潜水员, 变压性眩晕

\section{1. 引言}

1957年美国海军潜水生理学家Bond提出饱和潜水概 念, 提高了潜水作业效率, 但由于氮麻醉的影响, 深度大 于 $36.5 \mathrm{~m}$ 的饱和潜水应用氦氧混合气, 称为“氦氧饱和潜 水”[1]。我国上世纪70年代起进行了一系列空气饱和以及 氮氧饱和潜水医学的研究, 80 年代后进行了一系列大深度 氦氧饱和潜水医学保障的研究, 从实验室模拟到海上实潜, 饱和深度从 $80 \mathrm{~m}$ 到 $350 \mathrm{~m}$, 取得饱和潜水对机体生理影响以 及医学保障的研究成果 $[2,3]$ 。

1965年, Lundgren[4]对550名潜水员的调查研究中首 次命名了“变压性眩晕”, 指外界压力变化时急性发作的旋 转性眩晕。飞行员在飞行过程中也存在压力变化的, 对于 飞行员的变压性眩军的研究较多 [5,6], 而对于潜水员的研 究报道则较少, 随着人类向深海探索的需要, 更大深度饱 和潜水的医学研究是重要的课题。某研究所在 $500 \mathrm{~m}$ 饱和 潜水系统进行了深度递增模拟 $65 \mathrm{~m} 、 250 \mathrm{~m} 、 480 \mathrm{~m}$ 氦氧饱 和潜水实验[7], 笔者作为临床保障医师进行了相关调查, 现将结果进行报道。为越来越多的常规潜水以及饱和潜水 作业提供临床医学保障知识, 能够使更多的潜水医师认识 和诊断此病, 并能够准确处理, 可进一步保障潜水员健康, 提高潜水作业效率, 并为进一步加深饱和潜水实验提供参 数。

\section{2. 研究对象与方法}

\section{1. 研究对象}

9名潜水员, 年龄 $25 \sim 34$ 岁, 潜龄 $6 \sim 16$ 年。饱和潜水 实验在某医学研究所 $500 \mathrm{~m}$ 饱和潜水系统内进行。潜水员 身体及心理均健康, 无眩晕相关疾病病史, 选拔情况见文 献[7]。

\section{2. 方法}

\subsection{1. 深度递增模拟模拟氦氧饱和潜水方法}

深度递增模拟分 3 个阶段 5 次饱和潜水组成, 分别为 $65 \mathrm{~m}$ (2次): 加压 $2 \mathrm{~h}$, 饱和逗留 $48 \mathrm{~h}$, 减压 $52 \mathrm{~h} ; 250 \mathrm{~m}$ (2次): 加压 $13 \mathrm{~h}$, 饱和逗留 $47 \mathrm{~h}$, 减压 $178 \mathrm{~h}$; 和 $480 \mathrm{~m}$ (1次): 加压 82h, 480m逗留-493m巡回49h, 减压302h。潜水具体过程 参阅文献[7]。

\subsection{2. 体检及检查}

每次饱和潜水前 1 周以及出舱后第 $2 \mathrm{~d}$ 和 1 月均住院检 查, 进行神经科以及耳鼻喉科副主任医师以上医师体检, 发现异常则另一名主任医师复检。除常规查体外, 神经内 科进行Romberg试验以及Fukuda试验, 耳鼻喉科进行电耳 镜检查、电测听以及前庭功能检查: 采用丹麦尔听美查特 眼震视图仪进行检测, 包括自发性眼震、变位试验和冷热 试验。潜水前后行头颅以及内耳MRI检查。

\subsection{3. 潜水时眩晕症状记录及检查}

潜水过程中时如果出现以下症状及时报告并填表: 天 旋地转、耳闷、耳狵、耳鸣、摇晃感、恶心、头昏、头痛, 产生症状的持续时间、潜水深度, 加压时间、减压时间等。 在舱内完成Romberg试验以及Fukuda试验, 记录是否正常。 如1名潜水员出现症状时,所有同舱潜水员均完成Romberg 试验以及Fukuda试验用以对照。

\subsection{4. 变压性眩晕诊断标准}

参阅文献 $[5,6]$ 制定标准: 条件:1、环境压力变化时或 存在等压气体交换时发生; 2 、出现 1 种及 1 种以上主要症 状: 天旋地转、耳闷、耳狵、耳鸣、摇晃感, 伴有或不伴 有恶心、头昏、头痛; 3、体检至少具备以下 1 种阳性体征: Romberg试验, Fukuda试验, 自发性眼震, 变位试验, 冷 热试验阳性; 4、头㐿MRI检查阴性。确诊标准为具备条 件 $1+2+3+4$, 极可能标准为具备条件 $1+2$, 或 $1+3+4$, 排除标 准为不具条件 1 和 4 。

\section{3. 结果}

\section{1. 一般资料及体检结果}

因为潜水员经过严格选拔，因此无高血压、糖尿病、 高血脂等病史, 无耳鼻喉慢性病史, 无中耳炎史, 无眩晕 病史, 无神经科疾病史, 无头部外伤史, 无精神疾病史, 心理评定健康。内外科检查均正常, 出舱后以及出舱 1 月 后查体均正常。 


\section{2. 神经科查体及头颎MRI}

$250 \mathrm{~m} 、 480 \mathrm{~m}$ 出舱后各有 1 名潜水员嗅觉减退, $250 \mathrm{~m}$ 、 $480 \mathrm{~m}$ 出舱后各有 1 名潜水员膝反射 $(+)$, 但是双侧对称,
$250 \mathrm{~m} 、 480 \mathrm{~m}$ 出舱后各有 1 名潜水员指鼻试验 $(+) ， 480 \mathrm{~m}$ 出舱后有 1 名潜水员跟胫膝实验 $(+)$, 其余查体及头颖 MRI异常率为 $0 \%$ 。所有异常者第二天复查均正常。见表 1 。

表1 神经科查体及头领MRI异常情况。

\begin{tabular}{|c|c|c|c|c|c|c|c|c|c|}
\hline & \multicolumn{3}{|c|}{$65 m$} & \multicolumn{3}{|c|}{$250 \mathrm{~m}$} & \multicolumn{2}{|r|}{480} & \multirow[b]{2}{*}{ 出舱1月 } \\
\hline & 前 & 出舱后 & 出舱1月 & 前 & 出舱后 & 出舱1月 & 前 & 出舱后 & \\
\hline 颅神经 & 0 & 0 & 0 & 0 & 1嗅觉减退 & 0 & 0 & 1嗅觉减退 & 0 \\
\hline 肌力 & 0 & 0 & 0 & 0 & 0 & 0 & 0 & 0 & 0 \\
\hline 肌张力 & 0 & 0 & 0 & 0 & 0 & 0 & 0 & 0 & 0 \\
\hline 腱反射 & 0 & 0 & 0 & 0 & $1(+)$ & 0 & 0 & $1(+)$ & 0 \\
\hline 巴氏征 & 0 & 0 & 0 & 0 & 0 & 0 & 0 & 0 & 0 \\
\hline 指鼻试验 & 0 & 0 & 0 & 0 & 1 & 0 & 0 & 1 & 0 \\
\hline Romberg & 0 & 0 & 0 & 0 & 0 & 0 & 0 & 1 & 0 \\
\hline Fukuda & 0 & 0 & 0 & 0 & 0 & 0 & 0 & 0 & 0 \\
\hline 头颅 MRI & 0 & 0 & 0 & 0 & 0 & 0 & 0 & 0 & 0 \\
\hline
\end{tabular}

\section{3. 耳鼻喉查体及内耳MRI}

$65 \mathrm{~m} 、 250 \mathrm{~m} 、 480 \mathrm{~m}$ 出舱后各有 1 名潜水员鼓膜充血, $250 \mathrm{~m}$ 出舱后 1 名潜水员, $480 \mathrm{~m}$ 出舱后 2 名潜水员冷热实验 (+), 均右侧, $250 \mathrm{~m} 、 480 \mathrm{~m}$ 出舱后各有 2 名潜水员电测 听示感音性受损，480m出舱后有 1 名潜水员跟膝胫实验 (+)，其余查体及内耳MRI异常率为 $0 \%$ 。所有异常者第 二天复查均正常。见表2。

表2 耳鼻喉科查体及内耳MRI异常情况。

\begin{tabular}{|c|c|c|c|c|c|c|c|c|c|}
\hline & \multicolumn{3}{|c|}{$65 \mathrm{~m}$} & \multicolumn{3}{|c|}{$250 \mathrm{~m}$} & \multicolumn{2}{|r|}{480} & \multirow[b]{2}{*}{ 出舱1月 } \\
\hline & 前 & 出舱后 & 出舱1月 & 前 & 出舱后 & 出舱1月 & 前 & 出舱后 & \\
\hline 耳道耵聍 & 0 & 0 & 0 & 0 & 0 & 0 & 0 & 0 & 0 \\
\hline 鼓膜穿孔 & 0 & 0 & 0 & 0 & 0 & 0 & 0 & 0 & 0 \\
\hline 鼓膜内陷 & 0 & 0 & 0 & 0 & 0 & 0 & 0 & 0 & 0 \\
\hline 鼓膜充血 & 0 & 1 & 0 & 0 & 1 & 0 & 0 & 1 & 0 \\
\hline 感音性损伤 & 0 & 0 & 0 & 0 & 2 & 0 & 0 & 2 & 0 \\
\hline 神经性损伤 & 0 & 0 & 0 & 0 & 0 & 0 & 0 & 0 & 0 \\
\hline 混合性损伤 & 0 & 0 & 0 & 0 & 0 & 0 & 0 & 0 & 0 \\
\hline 自发性眼震 & 0 & 0 & 0 & 0 & 0 & 0 & 0 & 0 & 0 \\
\hline 眼动功能 & 0 & 0 & 0 & 0 & 0 & 0 & 0 & 0 & 0 \\
\hline 变位试验 & 0 & 0 & 0 & 0 & 0 & 0 & 0 & 0 & 0 \\
\hline 冷热试验 & 0 & 0 & 0 & 0 & 1 & 0 & 0 & 2 & 0 \\
\hline 内耳MRI & 0 & 0 & 0 & 0 & 0 & 0 & 0 & 0 & 0 \\
\hline
\end{tabular}

\section{4. 眩晕症状统计结果}

高压停留阶段无压力变化, 症状不在统计中, 故天旋 地转发生 5 人次, 平均 $13 \mathrm{~S}$, 耳间发生 4 人次, 平均 $177.5 \mathrm{~s}$,
摇晃感发生 7 人次, 平均 $337.14 \mathrm{~s}$, 恶心 5 人次, 平均 $312 \mathrm{~s}$, 头昏 3 人次, 平均 $15 \mathrm{~min}$, 头痛 1 人次, $20 \mathrm{~s}$, Romberg6人次, Fukuda2人次, 症状发生情况统计结果, 见表3。

表3 眩晕症状。

\begin{tabular}{|c|c|c|c|c|c|c|c|c|c|}
\hline & \multicolumn{3}{|c|}{$65 \mathrm{~m}$} & \multicolumn{3}{|c|}{$250 \mathrm{~m}$} & \multicolumn{3}{|c|}{$480 \mathrm{~m}$} \\
\hline & 加压 & 高压停留 & 减压 & 加压 & 高压停留 & 减压 & 加压 & 高压停留 & 减压 \\
\hline 天旋地转 & 0 & 0 & 0 & $1,10 \mathrm{~s}$ & 0 & $1,15 \mathrm{~s}$ & $1,20 \mathrm{~s}$ & 0 & $2,30 \mathrm{~s}$ \\
\hline 耳闪 & $1,60 \mathrm{~s}$ & 0 & 0 & 0 & 0 & $1,110 \mathrm{~s}$ & 1,360 & $1,180 \mathrm{~s}$ & $1,180 \mathrm{~s}$ \\
\hline 耳袭 & 0 & 0 & 0 & 0 & 0 & 0 & 0 & 0 & 0 \\
\hline 耳鸣 & 0 & 0 & 0 & 0 & 0 & 0 & 0 & 0 & 0 \\
\hline 摇晃感 & 0 & 0 & $1,360 \mathrm{~s}$ & $2,300 \mathrm{~s}$ & 0 & $1,200 \mathrm{~s}$ & $1,600 \mathrm{~s}$ & $1,600 \mathrm{~s}$ & $2,900 \mathrm{~s}$ \\
\hline 恶心 & $1,300 \mathrm{~s}$ & 0 & 0 & 0 & $1300 \mathrm{~s}$ & $2,600 \mathrm{~S}$ & $1,360 \mathrm{~s}$ & $2,600 \mathrm{~s}$ & $1,300 \mathrm{~s}$ \\
\hline 头昏 & 0 & $1,20 \mathrm{~min}$ & 0 & 0 & $2,10 \mathrm{~min}$ & $1,15 \mathrm{~min}$ & 0 & $1,20 \mathrm{~min}$ & $2,15 \mathrm{~min}$ \\
\hline 头痛 & 0 & 0 & 0 & 0 & 0 & 0 & $1,20 \mathrm{~s}$ & 0 & 0 \\
\hline Romberg & 0 & 0 & 1 & 1 & 1 & 1 & 1 & 0 & 2 \\
\hline Fukuda & 0 & 0 & & 0 & 0 & 1 & 0 & 0 & 1 \\
\hline
\end{tabular}




\section{5. 变压性眩晕诊断结果}

$65 \mathrm{~m}$ 饱和潜水确诊 1 人次, 极可能 1 人次, $250 \mathrm{~m}$ 饱和潜水确诊 2 人次, 极可能 2 人次, $480 \mathrm{~m}$ 确诊 3 人次。

表4 变压性眩晕诊断情况。

\begin{tabular}{|c|c|c|c|c|c|c|c|c|c|}
\hline & \multicolumn{3}{|c|}{$65 \mathrm{~m}$} & \multicolumn{3}{|c|}{$250 \mathrm{~m}$} & \multicolumn{3}{|c|}{$480 \mathrm{~m}$} \\
\hline & 加压 & 高压停留 & 减压 & 加压 & 高压停留 & 减压 & 加压 & 高压停留 & 减压 \\
\hline 确诊 & 0 & 0 & 1 & 1 & 0 & 1 & 1 & 0 & 2 \\
\hline 极可能 & 1 & 0 & 0 & 1 & 0 & 1 & 0 & 0 & 0 \\
\hline
\end{tabular}

\section{4. 讨论}

自1965年提出变压性眩晕后在潜水员中的研究较少 $[8,9]$, 其发病率约 $26 \%$ 。而在飞行员中国内外研究多见, 国外报道约为 $10 \% \sim 17 \%[5]$, 金占国[6]调查发现国内歼击 机飞行员变压性眩晕的发生率为 $20.72 \%$, 并认为随着飞机 性能的不断提升, 变压性眩晕发生率亦可随之提高。本研 究共 9 人次发病, 因为非流行病调查研究, 样本较少, 并 且观察的情况为大深度氦氧饱和潜水, 存在 1 人多次潜水, 以及潜水过程多次发病的情况, 因此未做发病率计算, 但 是从发病确诊以及极可能情况看, 变压性眩晕不是少见疾 病, 应当引起重视。之所以目前未引起重视, 与这种眩晕 为一过性, 恢复快, 未造成实质性损伤, 以及大家认识不 够等因素有关 $[10,11]$ 。本研究中神经科以及耳鼻喉科查体 结果有个例出现嗅觉、腱反射减退, 以及共济失调, 鼓膜 充血, 听力、前庭功能受损, 但是自己无症状, 查体时发 现, 第二天复查均正常, 说明程度轻, 发生过程中往往自 己克服, 潜水后一般也不会提及, 因此均不予重视。

变压性眩晕未见诊断标准[12], 本研究制定了初步标 准: 条件1、肯定环境压力变化时或存在等压气体交换时, 2、出现主要症状: 天旋地转、耳闷、耳狵、耳鸣、摇晃 感一种, 伴有或不伴有恶心、头昏、头痛; 3 、体检具备 1 种及1种以上阳性体征: Romberg试验, Fukuda试验, 自发 性眼震, 变位试验, 冷热试验阳性, 4、头颖MRI检查阴 性。确诊标准为具备条件 $1+2+3+4$, 极可能标准为具备条 件 $1+2$,或 $1+3+4$, 排除标准为不具条件 1 和 4 。从 3 个阶段的 潜水过程中出现症状及体征结果来看, 这个标准是可行的。 MRI的检查需要大型设备, 虽然现在越来越普及, 但是也 存在不具备MRI的检查的医疗机构, 因此这种情况只能诊 断极可能, 有条件时必须完善MRI检查。

飞行员的变压性眩晕研究中, 发现许多诱发因素 [13 15], 例如: 内耳的潜在性疾病, 一侧前庭功能异常、 潜在的梅尼埃病、迷路炎、感冒未愈、急性鼻炎、以及面 罩吸氧等因素, 本研究中未发现这类情况, 因为本次大深 度潜水实验, 潜水员严格选拔, 潜水员全面体检, 选择出 非常合格的潜水员, 排除了急、慢性病史的潜水员, 但是 在平时的潜水中, 不排除上述因素诱发潜水员发生变压性 眩晕的可能, 因此这些诱发因素也应予以重视。

\section{5. 结论}

综上所述, 大深度氦氧饱和潜水变压性眩晕多人次发 生, 特点是时间短, 完全可逆, 未对潜水员健康造成影响,
但是应引起重视。本文提出的诊断标准实用性强, 易于推 广应用, 适合于各级医疗机构。

\section{参考文献}

[1] 方以群,王敏,陈伯华.饱和潜水及其医学保障(上)[J].人民军 医2013,56(11):1278-1279,1285。

[2] 龚锦涵,罗锐仁, 案印宝, 等. $350 \mathrm{~m}$ 模拟氦氧 饱和 $-370 \mathrm{~m}$ 巡回潜 水实 验研究 [J]. 海 洋工程 1991,9(3):98-103。

[3] 石中瑗,赵德铭, 崔人镜, 等. 302米氦 氧饱和潜 水模 拟实验 中人体某些生理功能的观察[J].海洋工 程, 1983,1(14):74-83。

[4] Lundgren CE. Alternobaric vertigo--a diving hazard[J]. Br M ed J,1965,2(5460):511-513。

[5] Subtil J,V arandas J,G alrão F,et al. A lternobaric vertigo:prevalence in Portuguese Air Force pilots[J].Acta Otolaryngol,2007, 127(8):843-846。

[6] 金占国,徐先荣,王健, 等. 歼击机飞行员变压性眩晕的调查 及相关因素分析 [J].解放军医学院学报2015,36(1):21-23。

[7] 肖卫兵,张民,方以群. 模拟氦氧 $480 \mathrm{~m}$ 饱和-493 $\mathrm{m}$ 巡回潜水 研究中华航海医学与高气压医学杂志 [J].2014,21(2):73-75,97。

[8] Bender-Heine Adam; Dillard Zachary W; Zdilla Matthew J; Alternobaric vertigo and facial baroparesis caused by scuba diving and relieved by chewing pineapple: a case report[J]. Undersea \& hyperbaric medicine: journal of the Undersea and Hyperbaric Medical Society, Inc 2017,44(6): 607-610。

[9] Jonathan R. Mallen MD, Daniel S. Roberts MD, PhD. SCUBA Medicine for otolaryngologists: Part I. Diving into SCUBA physiology and injury prevention[J]. The Laryngoscope, 2020, 130(1):52-58。

[10] Kitajima Naoharu, Sugita-Kitajima Akemi, Kitajima Seiji. Superior canal dehiscence syndrome associated with scuba diving[J]. Diving and hyperbaric medicine. 2017, 47(2):123-126。

[11] Endara-Bravo Andres, Ahoubim Daniel, Mezerhane Edward, et al. Alternobaric vertigo in a patient on positive airway pressure therapy.[J]. Journal of clinical sleep medicine: JCSM: official publication of the American Academy of Sleep Medicine, 2013, 9(12): 1347-8。 
[12] Ö.Tjernström. Further Studies on Alternobaric Vertigo: Posture and Passive Equilibration of Middle Ear Pressure[J]. Acta Oto-Laryngologica, 2009, 78(1-6): 1-231。

[13] Charles D. Bluestone, J. Douglas Swarts, Joseph M. Furman, et al. Persistent alternobaric vertigo at ground level[J]. The Laryngoscope, 2012, 122(4): 868-872。

[14] Evens Rachel A, Bardsley Barry, C Manchaiah Vinaya K. Auditory complaints in scuba divers: an overview.[J]. Indian journal of otolaryngology and head and neck surgery: official publication of the Association of Otolaryngologists of India, 2012, 64(1): 71-8。

[15] Tran Dai A. You're the flight surgeon: alternobaric vertigo.[J]. Aviation, space, and environmental medicine, 2010, 81(9): 896-7。 